\title{
Concentración de metales pesados en hongos de la zona metropolitana de la Comarca Lagunera, México
}

\section{Heavy metals concentration in fungi in the metropolitan area of the Comarca Lagunera, Mexico}

\author{
Lilia Iveth Domínguez-Zúñiga 1, Cristo Omar Puente-Valenzuela 2, Josué Raymundo Estrada-Arellano 1, \\ Elvira Aguirre-Acosta ${ }^{3}$, David Ramiro Aguillón-Gutiérrez ${ }^{4}$ \\ ${ }^{1}$ Facultad de Ciencias Biológicas, Universidad Juárez del Estado de Durango. Av. Universidad S/N, Fraccionamiento Filadelfia, \\ C.P. 35010, Gómez Palacio, Durango, México. \\ ${ }^{2}$ Centro Nacional de Investigación Disciplinaria en Relación Agua, Suelo, Planta, Atmósfera. Parque Industrial II, C.P. 34079, Gómez \\ Palacio, Durango, México. \\ ${ }^{3}$ Departamento de Botánica, Instituto de Biología, Universidad Nacional Autónoma de México. Tercer circuito exterior s/n, Ciudad \\ Universitaria, Coyoacán, C.P. 04510, Ciudad de México, México. \\ ${ }^{4}$ Laboratorio de Bioindicadores, Centro de Investigación y Jardín Etnobiológico, Universidad Autónoma de Coahuila. Dr. Francisco \\ González 37, C.P. 27480, Viesca, Coahuila, México.
}

\section{RESUMEN}

Antecedentes: A nivel mundial se han realizado varios estudios relacionados con macrohongos y metales pesados, pero en México existen pocos registros al respecto. Anteriormente en la zona de la Comarca Lagunera se han registrado niveles de metales pesados en diversos organismos.

Objetivos: Este trabajo tiene como objetivo determinar la concentración de metales pesados ( $\mathrm{Cd}, \mathrm{Cu}, \mathrm{Pb}$ y $\mathrm{Zn}$ ) en especies fúngicas en la zona metropolitana de la Comarca Lagunera, México.

Métodos: Se realizaron dos recolectas anuales (2017-2018) en 30 sitios pertenecientes a principales áreas verdes y avenidas de tres municipios de la Comarca Lagunera. La identificación de los ejemplares se realizó mediante técnicas de micología básica y literatura especializada. En cuanto a la medición de metales se empleó la espectroscopía de absorción atómica con aspiración directa.

Resultados y conclusiones: Se obtuvieron un total de 32 muestras, pertenecientes a nueve especies. Los resultados mostraron concentraciones altas en las nueve especies analizadas, sobrepasando los parámetros de límites permisibles por estándares europeos.

Palabras clave: macrohongos, zona urbana, bioindicadores, contaminación, espectroscopía

\section{ABSTRACT}

Background: Globally several studies related to macrofungi and heavy metals have been carried out worldwide, but in Mexico there are few records in this regard. Previously in the Lagunera Region, levels of heavy metals have been recorded in various organisms.

Objectives: This work aims to determine the concentration of heavy metals $(\mathrm{Cd}, \mathrm{Cu}, \mathrm{Pb}$ and $\mathrm{Zn})$ in fungal species in the metropolitan area of the Lagunera Region, Mexico.

Methods: Two annual collections were carried out (2017-2018) in 30 sites belonging to the main green areas and avenues of three municipalities of the Lagunera Region. The identification of the species was made according to basic mycological methods and specialized literature. Regarding the reading of metals, atomic absorption spectroscopy with direct aspiration was used.

Results: A total of 32 samples were obtained, belonging to nine species. The results showed high concentrations in the nine species analyzed, exceeding the parameters of permissible limits by European standards.

Keywords: macrofungi, urban zone, bioindicators, pollution, spectroscopy

\section{ARTICLE HISTORY}

Received 29 November 2020 / Accepted 17 May 2021

Published on line: 20 June 2021

\section{CORRESPONDING AUTHOR}

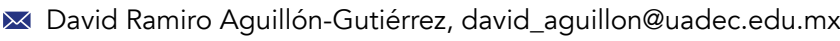
ORCID: 0000-0002-2519-8928 


\section{INTRODUCCIÓN}

Los metales pesados son sustancias propias de la naturaleza de peso molecular alto; tienen efectos en la salud y afectan a diferentes órganos en humanos. Estos metales pueden provocar cefaleas, daños en el sistema neurológico, lesiones a nivel celular, insuficiencia renal y cáncer (Eróstegui 2009). La actividad minera genera grandes cantidades de desechos que contienen altas concentraciones de metales, los cuales son una fuente de contaminación aun después de haber cesado las actividades de extracción en un largo período (García-Sánchez et al. 1999). Actividades como la minería están relacionadas a contaminación del suelo afectando la biota y alterando los ciclos biogeoquímicos (Wong 2003).

Desde hace décadas se sabe que algunas especies de hongos acumulan en gran medida metales pesados tóxicos, como el plomo, mercurio y cadmio; los estudios de contenido de metales en hongos han demostrado una correlación entre la cantidad de metal en estos organismos y las fuentes de contaminación como las fundiciones y la proximidad a carreteras (Carvalho et al. 2005). Los esporocarpos son utilizados como bioindicadores para la contaminación ambiental por su corto período de vida (1-2 semanas), ya que pueden acumular minerales dependiendo generalmente de su metabolismo, el cual es afectado por la composición química del sustrato, del cual los hongos obtienen sus nutrientes (Garnweidner 1992, Radulescu et al. 2010). Los hongos poseen mecanismos para la unión y remoción de metales desde interacciones fisicoquímicas en la superficie celular como la absorción, hasta procesos de metabolismo celular como la precipitación extracelular del metal, lo cual ha permitido la supervivencia de especies fúngicas ante la toxicidad de los metales. Las interacciones hongo-metal pueden suceder de varias maneras, dependiendo del tipo de metal, ejerciendo sus efectos en el bloqueo funcional de enzimas (Gutiérrez-Corona et al. 2007, Anahid et al. 2011). La captación de metales y otros elementos depende de una serie de factores medio ambientales y del propio hongo, incluyendo entre estos el transporte atmosférico de polvo y sedimentos del suelo a través de flujos y formas fluviales, además de la presencia de grupos funcionales tales como el fosfato, carboxil y amino, mismos que permiten la capacidad de acumulación de metales pesados (Schmitt y Meisch 1985, Turnau et al. 1994, Alonso et al. 2010, Cadavid-Velásquez et al. 2019).

En un estudio realizado por Benin et al. (1999) se demostró que en Torreón hay plomo y cadmio en niveles similares a los de otros sitios contaminados del mundo y superiores a los que establece la Agencia de Protección Ambiental de Estados Unidos (EPA) para considerarlos seguros. El estudio indicó la existencia de niveles de plomo que iban desde 787 hasta 13, 231 rg/g; para cadmio las concentraciones en el polvo variaban entre los 11 y 1, $497 \mu \mathrm{g} / \mathrm{g}$. Dado que el plomo y el cadmio entran al organismo por la ingesta de polvo, el estudio adquiere una importancia central para evaluar las dimensiones del problema y para establecer estrategias de remediación (Valdés y Morelos 1999, García-Vargas et al. 2007). Este trabajo tiene como objetivo determinar la concentración de metales pesados ( $\mathrm{Cd}, \mathrm{Cu}, \mathrm{Pb}$ y $\mathrm{Zn}$ ) en especies fúngicas en la zona metropolitana de la Comarca Lagunera, México.

\section{MATERIALES Y MÉTODOS}

\section{Zona de estudio}

La Comarca Lagunera está ubicada en el norte-centro de México, integrada por cinco municipios del suroeste del estado de Coahuila y 10 del noreste del estado de Durango. En este estudio se trabajó con las especies fúngicas presentes en los municipios de Torreón en el estado de Coahuila y de Gómez Palacio y Lerdo en el estado de Durango. El clima es seco desértico con una precipitación media anual de 250-350 mm, con una altitud media de $1100 \mathrm{msnm}$. Sus principales actividades económicas son las operaciones mineras y actividades agropecuarias (Rosas et al. 1999, Orona-Castillo et al. 2006).

\section{Muestreo}

Se realizaron dos períodos de recolectas abarcando los meses de septiembre a octubre de 2017 y de enero a octubre de 2018, haciendo un muestreo oportunista en distintos puntos que conforman la zona metropolitana de la Comarca Lagunera en donde se eligieron las especies con mayor frecuencia y tamaño con el fin de obtener la biomasa requerida para los análisis correspondientes, y asimismo comparar la cantidad de concentración entre las especies colectadas. La recolecta de ejemplares se llevó a cabo en las principales áreas verdes y avenidas de la zona urbana. Para cada 
municipio fueron determinados 10 sitios de muestreo, siendo en total 30. Los sitios de recolecta fueron establecidos principalmente en las áreas verdes urbanas por su cercanía con industrias y calles con mayor tráfico vehicular, debido a que en esos sitios la probabilidad de que crezcan especies de macrohongos es mayor a causa de que la vegetación juega un rol importante para el desarrollo de estos.

\section{Preparación de las muestras}

Una vez recolectados los ejemplares, se realizó la descripción en fresco utilizando el formato propuesto por Castañeda (2011); las muestras fueron colocadas en papel aluminio y bolsas de papel; cada ejemplar fue etiquetado y fotografiado correspondientemente para luego ser depositado en un contenedor hermético, esto con la finalidad de mantener frescos los ejemplares hasta ser depositados en laboratorio; ya en laboratorio los ejemplares fueron deshidratados en secadora durante 48 horas a una temperatura de $40{ }^{\circ} \mathrm{C}$, y posteriormente fueron resguardados en cajas de cartón para su posterior identificación.

Para la identificación de los ejemplares recolectados, se llevó a cabo la descripción de caracteres a nivel macroscópico y microscópico, utilizando las siguientes claves y literatura especializada: Cifuentes et al. (1984), Guzmán (1997), Michael et al. (2012), Valenzuela et al. (2013) y Cripps et al. (2016). Para la validación de los nombres de las especies, se utilizó el Index Fungorum (http://www.indexfungorum.org/names/names.asp).

Después de ser identificadas las muestras, se realizó una limpieza con una brocha con la finalidad de remover el exceso de tierra, luego las muestras fueron molidas en un molino de acero inoxidable; posteriormente se realizó un cribado a un tamaño de $0.5 \mathrm{~mm}$ de diámetro.

Se procedió a pesar $0.02 \mathrm{~g}$ de la biomasa obtenida; la digestión se efectuó con $\mathrm{HNO}_{3}$ al grado/trace select y peróxido de hidrógeno $\left(\mathrm{H}_{2} \mathrm{O}_{2}\right)$ al $30 \% \mathrm{P} / \mathrm{V}$, utilizando $400 \mathrm{~mL}$ de cada uno, además se agregaron $200 \mathrm{~mL}$ de agua milli-Q por 48 horas.

\section{Análisis}

El método empleado para la lectura de metales fue espectroscopía de absorción atómica con aspiración directa de acuerdo a los métodos EPA respectivos (EPA 1978a, 1978b, 1978c). Se utilizaron cinco estándares para cada metal (plomo, cadmio, cobre y zinc) con un coeficiente de correlación $>0.995$.

\section{RESULTADOS}

Se obtuvieron 32 muestras pertenecientes a nueve especies: Chlorophyllum molybdites (G. Mey) Massee, Inonotus aff. farlowii, Inonotus aff. hispidus, Podaxis pistillaris (L.) Fr., Agaricus sp., Ganoderma sp., Coprinopsis lagopus (Fr.) Redhead, Vilgalys \& Moncalvo, Leucocoprinus birnbaumii (Corda) Singer y Coprinellus truncorum (Scop.) Redhead, Vilgalys \& Moncalvo. Las especies fueron encontradas principalmente sobre avenidas, camellones y áreas verdes pertenecientes a la zona metropolitana de la Comarca Lagunera (Figura 1).

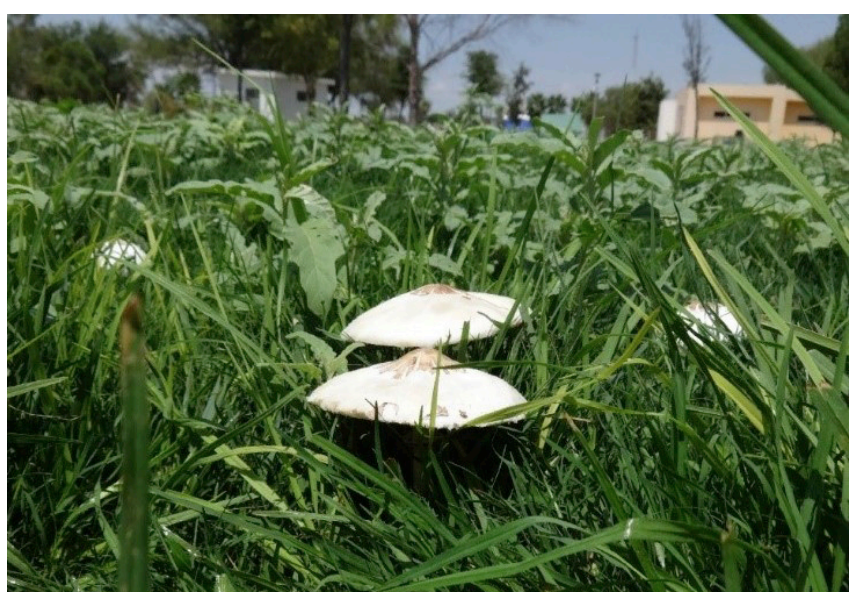

Figura 1. Ejemplares de Chlorophyllum molybdites encontrados en jardín urbano en el municipio de Torreón, Coahuila.

Las concentraciones de los metales analizados se muestran en la Tabla 1, en donde se puede observar que la mayoría de los ejemplares fueron encontrados en el municipio de Gómez Palacio. En cuanto a metales, el valor más alto para $\mathrm{Cd}$ y $\mathrm{Zn}$ se encontró en Coprinellus truncorum con $16.35 \mathrm{mg} / \mathrm{kg}$ y $420.98 \mathrm{mg} /$ kg respectivamente; en $\mathrm{Cu}$ Chlorophyllum molybdites obtuvo una concentración de $174.23 \mathrm{mg} / \mathrm{kg}$, para $\mathrm{Pb}$ se puede observar que en la mitad de los ejemplares no se detectó este metal, y su concentración máxima se observó en Ganoderma sp. con 147.3 mg/kg.

De los cuatro metales analizados en este trabajo se obtuvieron los promedios por especie, donde C. molybdites obtuvo el promedio más alto de $\mathrm{Cd}, \mathrm{Cu}$ y $\mathrm{Zn}$; mientras que Ganoderma sp. obtuvo el mayor prome- 
dio en $\mathrm{Pb}$ (Figura 2). Las concentraciones de $\mathrm{Cd}$, Cu y $\mathrm{Pb}$ encontradas en las especies fúngicas sobrepasan los parámetros de límites permisibles por estándares europeos (Reglamento CE 2006, 2007); para México no se encontró ninguna normativa que regule metales en macromicetos, por lo cual se consideró la NOM130-SSA1-1995 (Tabla 2).
En cuanto a los municipios, Torreón fue el que presentó una concentración alta de contaminación por metales pesados en especies fúngicas para $\mathrm{Pb}$ y $\mathrm{Zn}$, seguido por Lerdo con valores altos en $\mathrm{Cu}, \mathrm{Cd}$ y $\mathrm{Zn}$, mientras que Gómez Palacio presentó concentraciones variables (Figura 3).

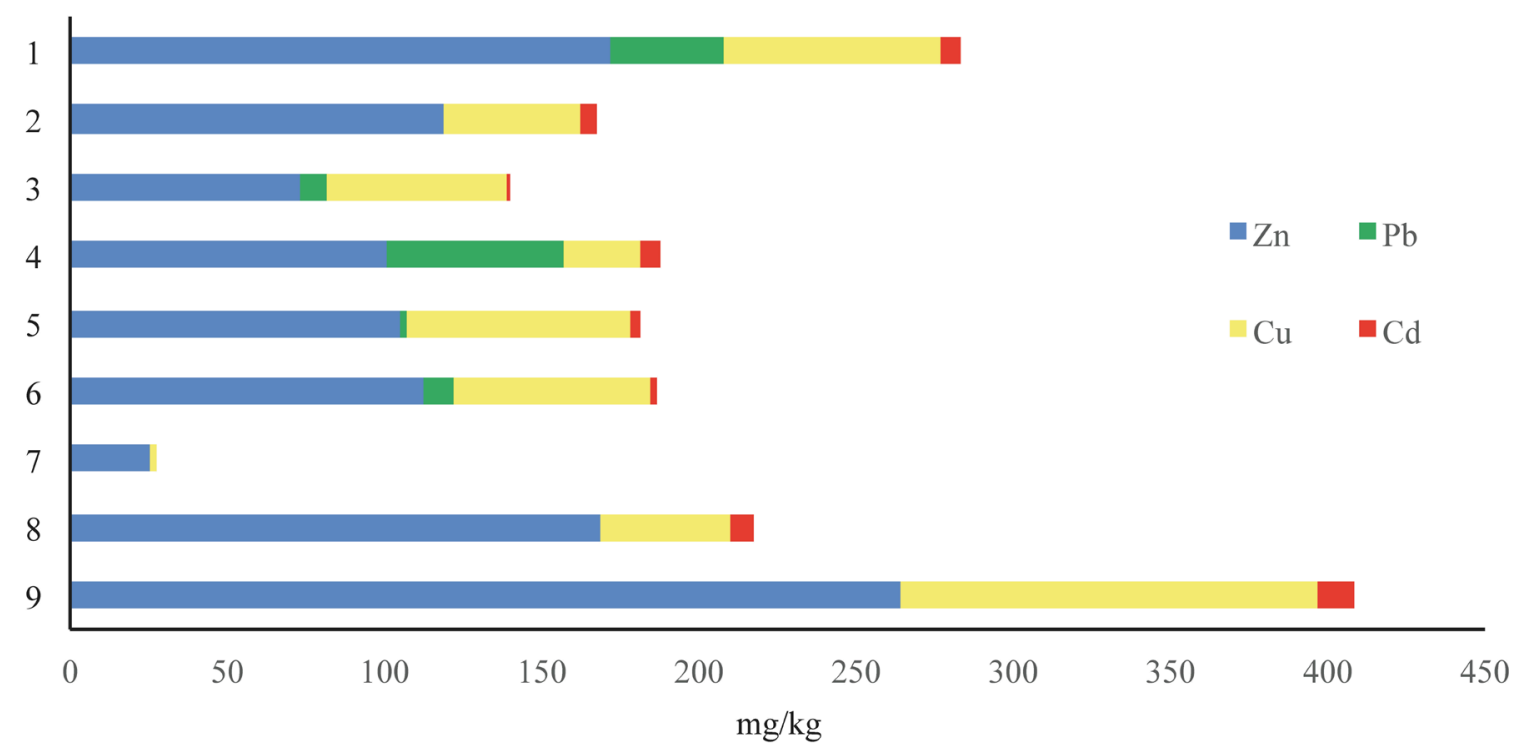

Figura 2. Promedios de las concentraciones de metales en las especies analizadas. 1: Coprinellus truncorum. 2: Leucocoprinus birnbaumii. 3: Coprinopsis lagopus. 4: Ganoderma sp. 5: Agaricus sp. 6: Podaxis pistillaris. 7: Inonotus. aff. hispidus. 8: Inonotus aff. farlowii. 9: Chlorophyllum molybdites.

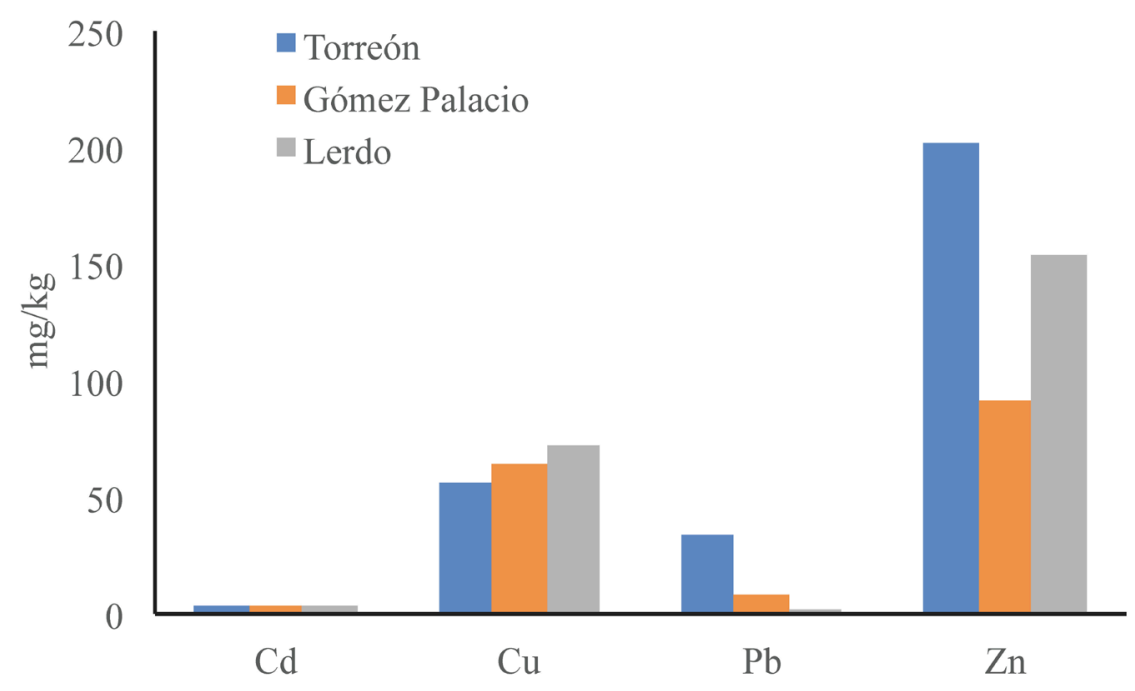

Figura 3. Promedios de las concentraciones de metales en las especies encontradas por municipio. 
Tabla 1. Especies, localización y concentración de metales $(\mathrm{mg} / \mathrm{kg})$ en las muestras analizadas

\begin{tabular}{|c|c|c|c|c|c|c|}
\hline Especie & Municipio & Muestra & $\mathrm{Cd}$ & $\mathrm{Cu}$ & $\mathrm{Pb}$ & $\mathrm{Zn}$ \\
\hline Agaricus sp. & GP & 8 & 5.65 & 68.69 & $N D^{*}$ & 96.84 \\
\hline Agaricus sp. & GP & 11 & 0.23 & 122.23 & 5.11 & 264.14 \\
\hline Agaricus sp. & GP & 15 & 8.44 & 53.25 & ND & 107.3 \\
\hline Agaricus sp. & GP & 23 & 0.23 & 24.93 & ND & 16.68 \\
\hline Agaricus sp. & GP & 26 & 1 & 46.3 & ND & 72.45 \\
\hline Agaricus sp. & GP & 30 & ND & 78.99 & 8.52 & 93.36 \\
\hline Agaricus sp. & $\mathrm{L}$ & 4 & ND & 82.34 & ND & 72.45 \\
\hline Agaricus sp. & L & 13 & 5.65 & 89.54 & 3.41 & 112.53 \\
\hline Chlorophyllum molybdites & $\mathrm{T}$ & 2 & 10.15 & 131.76 & ND & 246.72 \\
\hline Chlorophyllum molybdites & $\mathrm{T}$ & 22 & ND & 91.86 & ND & 368.7 \\
\hline Chlorophyllum molybdites & $\mathrm{L}$ & 25 & 14.18 & 174.23 & ND & 177.01 \\
\hline Coprinellus truncorum & GP & 6 & 5.03 & 75.13 & ND & 42.82 \\
\hline Coprinellus truncorum & GP & 9 & 16.35 & 68.69 & 3.41 & 211.86 \\
\hline Coprinellus truncorum & GP & 31 & 0.69 & 50.67 & 100.49 & 11.45 \\
\hline Coprinellus truncorum & $\mathrm{L}$ & 16 & 6.12 & 81.56 & 3.41 & 420.98 \\
\hline Coprinopsis lagopus & GP & 10 & 0.23 & 66.12 & 1.7 & 11.9 \\
\hline Coprinopsis lagopus & GP & 29 & ND & 48.1 & 15.33 & 133.44 \\
\hline Ganoderma sp. & $\mathrm{T}$ & 17 & 1 & 31.63 & 11.92 & 72.45 \\
\hline Ganoderma sp. & $\mathrm{T}$ & 27 & ND & 24.42 & 147.3 & 63.73 \\
\hline Ganoderma sp. & $\mathrm{T}$ & 32 & 11.7 & 33.94 & 8.52 & 239.74 \\
\hline Ganoderma sp. & $\mathrm{L}$ & 20 & ND & 18.5 & ND & 25.39 \\
\hline Ganoderma sp. & $\mathrm{L}$ & 21 & ND & 15.67 & ND & 103.81 \\
\hline Inonotus aff. farlowii & L & 7 & 0.07 & 41.66 & ND & 168.29 \\
\hline Inonotus aff. hispidus & GP & 24 & ND & 1.77 & ND & 25.41 \\
\hline Leucocoprinus birnbaumii & GP & 5 & 7.05 & 76.42 & ND & 229.29 \\
\hline Leucocoprinus birnbaumii & GP & 28 & 1.78 & 12.06 & ND & 7.63 \\
\hline Podaxis pistillaris & GP & 1 & 0.07 & 7.75 & $N D^{*}$ & 67.22 \\
\hline Podaxis pistillaris & GP & 3 & ND & 136.91 & 6.81 & 89.87 \\
\hline Podaxis pistillaris & GP & 12 & 3.33 & 84.14 & 2.55 & 63.73 \\
\hline Podaxis pistillaris & GP & 14 & 3.95 & 99.07 & 3.41 & 116.01 \\
\hline Podaxis pistillaris & GP & 18 & 2.09 & 97.01 & 2.55 & 72.45 \\
\hline Podaxis pistillaris & $\mathrm{T}$ & 19 & 0.69 & 24.93 & 32.36 & 220.58 \\
\hline
\end{tabular}

ND: no detectado. T: Torreón, Coahuila. GP: Gómez Palacio, Durango. L: Lerdo, Durango 
Tabla 2. Comparación entre los límites permisibles de metales en hongos en diferentes reglamentos y valores encontrados en este trabajo

\begin{tabular}{|c|c|c|c|}
\hline Norma/reglamento & Metal & Límites permisibles (mg/kg) & $\begin{array}{c}\text { Valores en este trabajo } \\
(\mathrm{mg} / \mathrm{kg})\end{array}$ \\
\hline Reglamento No 1881/2006 Comisión de las Comunidades Europeas & $\mathrm{Cd}$ & 0.050 & 16.35 \\
\hline Reglamento 333/ 2007 Comisión de las Comunidades Europeas & & 1.0 & \\
\hline NOM-130-SSA1-1995 & & 0.1 & \\
\hline NOM-130-SSA1-1995 & $\mathrm{Cu}$ & 5.0 & 174.23 \\
\hline Reglamento No 1881/2006 Comisión de las Comunidades Europeas & $\mathrm{Pb}$ & 0.10 & 147.3 \\
\hline Reglamento 333/ 2007 Comisión de las Comunidades Europeas & & 0.10 & \\
\hline NOM-130-SSA1-1995 & & 0.3 & \\
\hline NOM-130-SSA1-1995 & $\mathrm{Zn}$ & 5.0 & 420.98 \\
\hline
\end{tabular}

\section{DISCUSIÓN}

Las nueve especies analizadas en este trabajo presentaron concentraciones altas de metales pesados y por lo tanto potencial por concentrar estos elementos. En el municipio de Torreón, Coahuila, se encontraron las especies con mayor concentración de metales en $\mathrm{Pb}$ y Zn, seguido por Gómez Palacio, Durango. Entre las actividades económicas principales en ambas ciudades se encuentran la industria minera y la fundición de metales (Saavedra-Silva y Morales-Ramírez 2009), por lo que tienen un mayor nivel de contaminación, sin embargo no se pueden descartar otras fuentes de contaminación como el mal manejo de residuos y desechos, lo cual se ve reflejado en la presencia de metales pesados en los cuerpos fructíferos de los hongos; sin embargo el municipio de Lerdo presentó valores altos en $\mathrm{Cu}$, Zn y Cd, a diferencia de Gómez Palacio y Torreón. Lerdo es un municipio más pequeño poblacionalmente y con menor cantidad de industrias, por lo cual los valores altos que se presentaron en los metales mencionados pueden deberse a que estos elementos son fuentes importantes en los cuerpos fructíferos en especies de macrohongos. Además, como reportan Kokkoris et al. (2019) y Adebiyi y Adeyemi (2020), me- tales como plomo, cobre, manganeso, cromo, cadmio, hierro, zinc y níquel se bioacumulan en los cuerpos fructíferos de los hongos. Cabe destacar que el municipio de Lerdo cuenta con una estructura vegetal diferente, con una mayor cantidad de áreas verdes y además existe una menor urbanización, factor asociado a presencia de algunos de estos metales como $\mathrm{Cu}$ y $\mathrm{Zn}$ que tienen roles biológicos, además de que los hongos pudieran estar fungiendo como biorremediadores, al absorber los metales que se encuentran en el suelo (Das 2005). En el estudio realizado por Kalač (2009) se menciona que en el contenido usual de elementos en los cuerpos fructíferos de sitios no contaminados se pueden encontrar metales como: $\mathrm{Cu}, \mathrm{Cd}, \mathrm{Pb}, \mathrm{Mg}, \mathrm{As}$, $\mathrm{Ag}, \mathrm{Ni}$ y $\mathrm{Zn}$, variando los niveles dependiendo del sitio y lugar donde se desarrolla el basidioma.

La concentración máxima de $\mathrm{Cd}$ obtenida en este trabajo $(16.35 \mathrm{mg} / \mathrm{kg})$ difiere a lo encontrado por Aruguete et al. (1998), quienes reportan $4.09 \mathrm{mg} / \mathrm{kg}$, siendo esta cantidad considerablemente menor a la encontrada en esta investigación. Una situación similar ocurre con las concentraciones de $\mathrm{Cd}$ reportadas por Turkekul et al. (2004), en la ciudad de Tokat, Turquía, quienes reportan una concentración máxima de 1.8 $\mathrm{mg} / \mathrm{kg}$ para este metal; López-Vázquez y Prieto-Gar- 
cía (2016) reportaron una concentración máxima de 0.12 mg/kg; Quarcoo y Adotey (2013) reportan una concentración de $\mathrm{Cd}$ de $0.35 \mathrm{mg} / \mathrm{kg}$ en macrohongos provenientes de distintos mercados de Greater Accra en Ghana, siendo también una concentración considerablemente menor a la encontrada en este trabajo. Estas diferencias podrían deberse a que estos estudios fueron realizados en zonas boscosas, por lo cual no existe un factor de contaminación prevalente para que estas especies acumulen grandes cantidades de metales. Aunque, también existen reportes de altas cantidades de cadmio y otros metales en hongos silvestres de bosques cercanos a zonas industriales ( $\mathrm{Pa}$ jak, et al. 2020). También suponemos que las especies de flora juegan un papel importante en la captación de dichos metales, debido a que durante el estrés ambiental, las plantas exhiben una respuesta molecular, en donde abordan la toxicidad de los metales pesados al secuestrarlos en las vacuolas, sirviendo como almacenamiento temporal; a partir de esta capacidad las plantas son utilizadas para eliminar la contaminación ambiental por metales pesados mediante el proceso de fitorremediación (Salt et al. 1998). En cuanto a las concentraciones reportadas por Svoboda et al. (2000) en Eslovaquia oriental, Árvay et al. (2015) en Eslovaquia y Huang et al. (2015) en macrohongos de diversos mercados de Beijing, China (19.2, 18.0 y 13.8 mg/kg respectivamente), son similares a lo reportado en este trabajo, pero difieren de las reportadas por Alonso et al. (2004) y Zhu et al. (2010) (33.22 y 35.2 mg/kg respectivamente). Sarikurkcu et al. (2011) y Falandysz et al. (2018) registraron concentraciones de Cd (54.2 y 22 $\mathrm{mg} / \mathrm{kg}$ respectivamente) que sobrepasan a lo obtenido en esta investigación (16.35 mg/kg); cabe mencionar que ambos autores realizaron distintas técnicas de espectrofotometría (espectrofotometría de emisión óptica de plasma acoplada inductivamente).

En Cu se obtuvo una concentración máxima de 174.23 $\mathrm{mg} / \mathrm{kg}$ en este trabajo, siendo inferior a lo reportado por Svoboda et al. (2000) al Este de Eslovaquia y Alonso et al. (2004) en la Provincia de Lugo, España (505 y $212.5 \mathrm{mg} / \mathrm{kg}$ respectivamente); tal diferencia puede deberse a que el trabajo en Eslovaquia se llevó a cabo en un sitio de estudio que ha sido contaminado por emisiones de cobre y mercurio por parte de una industria local. En el caso de España el estudio se llevó a cabo utilizando otro método de cuantificación de metales, específicamente mediante voltamperometría de redisolución anódica de impulso diferencial para la lectura de dicho metal. El resultado de Cu obtenido en esta investigación (174.23 mg/kg) es superior a lo analizado por Paraskevi et al. (2009), Zhu et al. (2010), pero es similar a lo determinado por Sarikurkcu et al. (2011) (187 mg/kg).

En $\mathrm{Pb}$ las concentraciones más altas en este trabajo fueron de $100 \mathrm{mg} / \mathrm{kg}$ y $147.32 \mathrm{mg} / \mathrm{kg}$, siendo menor a lo reportado por Svoboda et al. (2000) con $223 \mathrm{mg} / \mathrm{kg}$ y superior a los análisis realizados por Siric et al. (2014), Huang et al. (2015), López-Vázquez y Prieto-García (2016). Estas diferencias pueden deberse a que los sitios de colecta se encontraban cercanos a industrias fundidoras de metal y próximos a carreteras con constante tráfico vehicular. Además, el resultado más alto de 147.32 mg/kg se encontró en Ganoderma sp., en el municipio de Torreón, ciudad que durante décadas ha tenido antecedentes de problemática ambiental relacionada al plomo (Flores y Albert, 2004). Debido a que Ganoderma sp. es una especie lignícola perenne (Pinzón-Osorio y Pinzón-Osorio 2016), probablemente ocurre un proceso de biomagnificación, sin embargo se requiere de mayores estudios sobre el proceso de biomagnificación en hongos y plantas para comprobar dicha hipótesis.

En cuanto a Zn, la concentración más alta en este trabajo fue de $420.98 \mathrm{mg} / \mathrm{kg}$, sobrepasando lo reportado por Isiloglu et al. (2001), Turkekul et al. (2004) y Sarikurkcu et al. (2011). Las diferencias pueden deberse a que estos estudios se realizaron en distintas regiones de Turquía, en donde se abarcaron las estaciones de otoño e invierno en tierras agrícolas sin antecedentes de contaminación. Para los estudios realizados por Paraskevi et al. (2009), Zhu et al. (2010), Mallikarjuna et al. (2013), Siric et al. (2014), Árvay et al. (2015), Falandysz et al. (2017) y Rasalanavho et al. (2019), las diferencias podrían deberse a los sitios de estudio, las cuales no presentan antecedentes de contaminación, además que las especies analizadas eran comestibles y a que se usaron metodologías de espectrofotometría distintas a las utilizadas en este trabajo. Sin embargo, las concentraciones reportadas por Alonso et al. (2004), Sesli et al. (2008), Huang et al. (2015), Falandysz et al. (2018) y Türkmen y Budur (2018) varian de 200 a 300 $\mathrm{mg} / \mathrm{kg}$, siendo menores a las reportadas en este trabajo $(420.98 \mathrm{mg} / \mathrm{kg})$.

De los resultados obtenidos se observa que el contenido metálico de las nueve especies que presentaron 
una acumulación que sobrepasa los límites permisibles de acuerdo a estándares europeos, genera una alerta en el consumo de especies comestibles, principalmente por especies silvestres. En México no existen normativas que regulen el contenido de metales en especies fúngicas, sin embargo, se consideró la siguiente norma: NOM-130-SSA1-1995, la cual especifica los límites permisibles de contenido metálico y microorganismos en alimentos envasados con cierre hermético y sometidos a tratamiento térmico; a pesar de esto, la norma no establece parámetros de límites permisibles de metales pesados en macrohongos silvestres, lo que crea un vacío en la legislación mexicana, ya que en distintas partes del país el consumo de hongos es habitual, como es en el caso de este trabajo en donde se encontraron los géneros Agaricus y Ganoderma, los cuales albergan especies comestibles y de valor medicinal, respectivamente.

\section{CONCLUSIONES}

Este trabajo demuestra que algunas especies de hongos macroscópicos (C. molybdites, P. pistillaris, Agaricus sp., Ganoderma sp., C. truncorum, I. aff. farlowii, I. aff. hispidus, C. lagopus y L. birnbaumii) presentes en la Comarca Lagunera (perteneciente a los estados de Coahuila y Durango, México) son capaces de acumular metales pesados ( $\mathrm{Cd}, \mathrm{Cu}, \mathrm{Pb}$ y $\mathrm{Zn}$ ) en cantidades superiores a las permitidas para alimentos por Normas Oficiales Mexicanas y por reglamentos europeos. Estos macrohongos desempeñan un papel importante en estos ecosistemas urbanos por su capacidad como bioindicadores en zonas contaminadas. Asimismo, no se encontraron publicaciones sobre la diversidad de macrohongos en el área urbana de La Comarca Lagunera, por lo cual consideramos a estas nueve especies como primer registro en la zona.

\section{AGRADECIMIENTOS}

El primer autor agradece al Instituto de Biología de la Universidad Nacional Autónoma de México, asimismo a las personas que ayudaron en este trabajo: Esaí Saldivar, Bun Alonso Saldaña, Yanaí Castor y Javier de la Fuente. Se reconoce el apoyo de la Universidad Juárez del Estado de Durango.

\section{LITERATURA CITADA}

Adebiyi AO, Adeyemi FP. 2020. Bioaccumulation of heavy metals in the fruiting bodies of four edible mushrooms collected from polluted areas in Akure, Ondo State, Nigeria. EAS Journal of Nutrition and Food Sciences 2, 19-23.

Alonso J, García M, Pérez-López J, Melgar M. 2004. Acumulación de metales pesados en macromicetos comestibles y factores que influyen en su captación. Revista de Toxicología 21, 11-15.

Alonso J, Fernández M, Melgar M, Pérez M, Corral M. 2010. Elementos traza en hongos comestibles. Repercusiones alimentarias y valoración nutricional. Boletín Micológico de FAMCAL 5, 101126.

Anahid S, Yaghmaei S, Ghobadinejad Z. 2011. Heavy metal tolerance of fungi. Scientia Iranica 18, 502-508. Doi: 10.1016/j. scient.2011.05.015

Aruguete DM, Aldstadt III JH, Mueller GM. 1998. Accumulation of several heavy metal and lanthanides in mushrooms (Agaricales) from the Chicago region. Sciencie of the Total Environment 224, 43-56. Doi: 10.1016/S0048-9697(98)00319-2

Árvay J, Tomáš J, Hauptvogl M, Massányi P, luboš H, Tóth T, Stanovič R, Bryndzová S, Bumbalová M. 2015. Human exposure to heavy metals and possible public health risks via consumption of wild edible mushrooms from Slovak Paradise National Park, Slovakia. Journal of Environmental Science and Health, Part B 50, 833843. Doi: 10.1080/03601234.2015.1058107

Benin AL, Sargent JD, Dalton M, Roda S. 1999. High Concentrations of heavy metals in neighborhoods near ore smelters in Northern Mexico. Environmental Health Perspectives 107, 279-284. Doi: 10.1289/ehp.99107279

Cadavid-Velásquez E, Pérez-Vásquez N, Marrugo-Negrete J. 2019. Contaminación por metales pesados en la bahía Cispatá en Córdoba-Colombia y su bioacumulación en macromicetos. Gestión y Ambiente 22, 43-53. Doi: 10.15446/ga.v22n1.75473

Carvalho ML, Pimentel AC, Fernández B. 2005. Study of heavy metal in wild edible mushrooms under different pollution conditions by X-Ray fluorescence spectrometry. Analytical Sciencies 21, 747750.

Castañeda LR. 2011. Manual para el Laboratorio de Micología. Facultad de Ciencias Biológicas, Universidad Juárez del Estado de Durango, Gómez Palacio.

Cifuentes BJ, Villegas RM, Pérez-Ramírez L. 1984. Claves para determinar macroscópicamente géneros de macromicetos. Herbario de la Facultad de Ciencias, UNAM, México, D.F.

Cripps C, Evenson V, Kou M. 2016. The essential guide to rocky mountain mushrooms by habitat. University of Illinois Press, Baltimore.

Das N. 2005. Heavy metals biosorption by mushrooms. Natural Product Radiance 4, 454-459.

EPA (Environmental Protection Agency). 1978a. Method 213.2: Cadmium. Atomic Absorption, furnace technique. The United States.

EPA (Environmental Protection Agency). 1978b. Method 239.2: Lead. Atomic Absorption, furnace technique. The United States. EPA (Environmental Protection Agency). 1978c. Method 289.2: Zinc. Atomic Absorption, Furnace Technique. The United States.

Eróstegui RC. 2009. Contaminación por metales pesados. Revista Científica Ciencia Médica 12, 45-46.

Falandysz J, Zhang J, Wiejak A, Baralkiewicz D, Hanc A. 2017. Metallic elements and metalloids in Boletus luridus, B. magnificus 
and B. tomentipes mushrooms from polymetallic soils from SW China. Ecotoxicology and Environmental Safety 142, 497-502. Doi: 10.1016/j.ecoenv.2017.04.055

Falandysz J, Medyk M, Treu R. 2018. Bio-concentration potential and associations of heavy metals in Amanita muscaria (L.) Lam. from northern regions of Poland. Environmental Science and Pollution Research 25, 25190-25206. Doi: 10.1007/s11356-018-2603-0

Flores J, Albert LA. 2004. Environmental lead in Mexico, 1990-2002. Reviews of Environmental Contamination and Toxicology 181, 37-109.

García-Sánchez A, Moyano A, Muñez C. 1999. Forms of cadmium, lead, and zinc in polluted mining soils and uptake by plants (Soria province, Spain). Commun. Soil Sciencie and Plant Analysis 30, 1385-1402. Doi: 10.1080/00103629909370294

García-Vargas GG, Rubio-Andrade M, Rosales-González MG, Goytia-Acevedo R, García-Arenas G, Candelas-Rangel JL, Meza-Velázquez R, Caravanos J. 2007. Contaminación por metales en suelos de la ciudad de Torreón, Coahuila, México. Revista Chapingo Serie Zonas Áridas 6, 165-168.

Garnweidner E. 1992. Setas. Como identificarlas, conocerlas y recogerlas. Ed. Everest S.A., León.

Gutiérrez-Corona F, Obregón-Herrera A, Cano-Canchola C. 2007. Resistencia a los metales pesados en hongos. In: Cervantes C., Moreno-Sánchez R.M. (eds.), Contaminación ambiental por metales pesados. Impacto en los seres vivos. Ed. A.G.T., México, D.F.

Guzmán G. 1997. Identificación de los hongos comestibles, venenosos y destructores de madera. Ed. Limusa, México, D.F.

Huang Q, Jia Y, Wan Y, Li H, Jiang R. 2015. Market survey and risk assessment for trace metals in edible fungi and the substrate role in accumulation of heavy metals. Journal of Food Science 80, 612-618. Doi: 10.1111/1750-3841.12923

Isiloglu M, Yilmaz F, Merdivan M. 2001. Concentrations of trace elements in wild edible mushrooms. Food Chemistry 73, 169-175. Doi: 10.1016/S0308-8146(00)00257-0

Kalač P. 2009. Chemical composition and nutritional value of European species of wild growing mushrooms: A review. Food Chemistry 113, 9-16. Doi: 10.1016/j.foodchem.2008.07.077

Kokkoris V, Massas I, Polemis E, Koutrotsios G, Zervakis GI. 2019. Accumulation of heavy metals by wild edible mushrooms with respect to soil substrates in the Athens metropolitan area (Greece). Science of the Total Environment 685, 280-296. Doi: 10.1016/j. scitotenv.2019.05.447

López-Vázquez E, Prieto-García F. 2016. Minerals and toxic elements in wild mushrooms species from regions of Hidalgo state in Mexico. Asian Journal of Chemistry 28, 2725-2730. Doi: 10.14233/ ajchem.2016.20098

Mallikarjuna SE, Ranjini, Devendra A, Haware J, Vijayalakshmi MR, Shashirekha MN, Rajarathnam S. 2013. Mineral composition of four edible mushrooms. Hindawi Publishing Corporation Journal of Chemistry 2013, 1-5. Doi: 10.1155/2013/805284

Michael RD, Sommer R, Menge JA. 2012. Field guide to mushrooms of western North America. University of California Prees, Berkeley.

Norma Oficial Mexicana (NOM-130-SSA1). 1995. Bienes y servicios. Alimentos envasados en recipientes de cierre hermético y so- metidos a tratamiento térmico. Disposiciones y especificaciones sanitarias. Diario Oficial de la Federación, México, D.F.

Orona-Castillo I, Espinoza-Arellano JJ, González-Cervantes G, Murillo-Amador B, García-Hernández JL, Santamaría CJ. 2006. Aspectos técnicos y socioeconómicos de la producción de nuez (Carya illinoensis Koch.) en la Comarca Lagunera, México. Agricultura Técnica en México 32, 295-301.

Pajak M, Gasiorek M, Jasik M, Halecki W, Otremba K, Pietrzykowski M. 2020. Risk assessment of potential food chain threats from edible wild mushrooms collected in forest ecosystems with heavy metal pollution in Upper Silesia. Poland. Forests 11, 12-40.

Paraskevi K, Petridis D, Dietrich Koller W, Riganakos K. 2009. Nutritional value and metal content of wild edible mushrooms collected from West Macedonia and Epirus, Greece. Food Chemistry 115, 1575-1580. Doi: 10.1016/j.foodchem.2009.02.014

Pinzón-Osorio CA, Pinzón-Osorio J. 2016. Ampliación de la distribución geográfica de Ganoderma australe (Patouillard, 1889) (Aphyllophorales-Basidiomycota), para el departamento de Cundinamarca, Colombia. Revista Biodiversidad Neotropical 1, 7-11.

Quarcoo A, Adotey G. 2013. Determination of heavy metals in Pleurotus ostreatus (Oyster mushroom) and Termitomyces clypeatus (Termite mushroom) sold on selected markets in Accra, Ghana. Mycosphere 5, 960-967.

Radulescu C, Stihi C, Busuioc G, Gheboianu Al, Popescu IV. 2010. Studies concerning heavy metals bioaccumulation of wild edible mushrooms from industrial area by using spectrometric techniques. Bulletin of Environmental Contamination and Toxicology 84, 641-646. Doi: 10.1007/s00128-010-9976-1

Rasalanavho M, Moodley R, Jonnalagadda S. 2019. Elemental distribution including toxic elements in edible and inedible wild growing mushrooms from South Africa. Environmental Science and Pollution Research 26, 7913-7925. Doi: 10.1007/s11356019-04223-0

Reglamento CE. 2006. Reglamento 1881/2006 de la Comisión de las Comunidades Europeas. Diario Oficial de la Unión Europea, Bruselas.

Reglamento CE. 2007. Reglamento 333/2007 de la Comisión de las Comunidades Europeas. Diario Oficial de la Unión Europea, Bruselas.

Rosas I, Belmont R, Armienta A, Baez A. 1999. Arsenic concentrations in water, soil, milk and forage in Comarca Lagunera, México. Water, Air, and Soil Pollution 112, 133-149. Doi: 10.1023/A:1005095900193

Saavedra-Silva EE, Morales-Ramírez J. 2009. Minería y metalúrgica. In: López-López A, Sánchez-Crispín A (eds.), Comarca lagunera: procesos regionales en el contexto global. Universidad Nacional Autónoma de México, México, D.F. Pp. 302-323.

Salt DE, Smith RD, Raskin I. 1998. Phytoremediation. Annual Review of Plant Physiology and Plant Molecular Biology 49, 643-668.

Sarikurkcu C, Copur M, Yildiz D, Akata I. 2011. Metal concentration of wild edible mushrooms in Soguksu National park in Turkey. Food Chemistry 128, 731-734. Doi: 10.1016/j.foodchem.2011.03.097

Schmitt JA, Meisch HU. 1985. Cadmium in mushrooms distribution, growth effects and binding. Journal of Trace Elements in Medicine and Biology 2, 163-166. 
Sesli E, Tuzen M, Soylak M. 2008. Evaluation of trace metal contents of some wild edible mushrooms from Black sea region, Turkey. Journal of Hazardous Materials 160, 462-467. Doi: 10.1016/j. jhazmat.2008.03.020

Siric I, Kos I, Bedekovic D, Kaic A, Kasap A. 2014. Heavy metals in edible mushroom Boletus reticulatus Schaeff. collected from Zrin mountain, Croatia. Periodicum Biologorum 3, 319-322.

Svoboda L, Zimmermannova K, Kalač P. 2000. Concentrations of mercury, cadmium, lead and copper in fruiting bodies of edible mushrooms in an emission area of a copper smelter and mercury smelter. The Sciencie of the Environment 246, 61-67. Doi: 10.1016/S0048-9697(99)00411-8

Turkekul I, Elmastasb M, Tuzenb M. 2004. Determination of iron, copper, manganese, zinc, lead, and cadmium in mushroom samples from Tokat, Turkey. Food Chemistry 84, 389-392. Doi: 10.1016/S0308-8146(03)00245-0

Türkmen M, Budur D. 2018. Heavy metal contaminations in edible wild mushroom species from Turkey's Black Sea region. Food Chemistry 254, 256-259. Doi: 10.1016/j.foodchem.2018.02.010
Turnau K, Kottke L, Dexheimer J, Botton B. 1994. Element distribution in mycelium of Pisolithus arrhizus treated with cadmium dust. Annals of Botany 74, 137-142. Doi: 10.1006/anbo.1994.1103

Valdés F, Morelos V. 1999. La contaminación por metales pesados en Torreón, Coahuila, México. Texas Center for Policy Studies-Ciudadanía Lagunera por los Derechos Humanos, Austin.

Valenzuela R, Raymundo T, Cifuentes J. 2013. El género Inonotus s. I. (Hymenochaetales: Agaricomycetes) en México. Revista Mexicana de Biodiversidad 84, 70-90. Doi: 10.7550/rmb.31605

Wong MH. 2003. Ecological restoration of mine degraded soils, with emphasis on metal contaminated soils. Chemosphere 6, 775780. Doi: 10.1016/S0045-6535(02)00232-1

Zhu F, Qu L, Fan W, Qiao M, Hao H, Wang X. 2010. Assessment of heavy metals in some wild edible mushrooms collected from Yunnan Province, China. Environmental Monitoring and Assessment 179, 191-199. Doi: 10.1007/s10661-010-1728-5. 